\title{
Single-incision laparoscopically assisted vaginal hysterectomy: Operative outcomes and its learning curve
}

\author{
TAKAHIRO KOYANAGI and SATORU MOTOMURA \\ Department of Obstetrics and Gynecology, Yanagawa Hospital, Yanagawa, Fukuoka, Japan \\ Received April 11,2011; Accepted May 13, 2011
}

DOI: $10.3892 /$ etm.2011.282

\begin{abstract}
We previously reported on single-incision laparoscopic surgery applied to laparoscopically assisted vaginal hysterectomy (LAVH) cases. We accumulated single-incision LAVH cases to evaluate this operation, including its learning curve. Since July 2009, we planned to perform single-incision LAVH in 50 cases. Operative time, estimated blood loss, weight of resected uterus and additional procedures were recorded and compared to those of conventional multiport, multi-incision LAVH. Additionally, 47 completed single-incision LAVH cases were divided into two groups; the former 24 cases and the latter 23 cases, to estimate its learning curve. Operative outcomes were statistically similar, except that more additional procedures were performed in the conventional LAVH group (27.7\% in single-incision vs. $57.5 \%$ in the conventional group, $\mathrm{P}<0.01)$. We experienced three conversions to multiport surgery from single-incision LAVH, and no conversion case to 'open' total abdominal hysterectomy from conventional LAVH, which was not significantly different $(3 / 50,6 \%$ vs. $0 / 40,0 \%, \mathrm{P}=0.12)$. During the study period, operative time was significantly shortened from $73.0 \pm 17.6 \mathrm{~min}$ for the former 24 cases to $58.0 \pm 12.2$ min for the latter 23 cases $(\mathrm{P}<0.01)$. There was no significant difference with respect to other operative outcomes between the two groups. Single-incision LAVH can be performed as effectively as conventional multiport LAVH with a short learning curve. We consider that single-incision LAVH may be a promising alternative method for the treatment of certain patients with uterine myomas and adenomyosis as even a less invasive gynecological operation is required without visible scars.
\end{abstract}

\section{Introduction}

Laparoscopically assisted vaginal hysterectomy (LAVH) has been widely accepted as a hysterectomy procedures for uterine

Correspondence to: Dr Takahiro Koyanagi, Department of Obstetrics and Gynecology, Yanagawa Hospital, 29 Chikushi-machi, Yanagawa-shi, Fukuoka 832-0077, Japan

E-mail: koyataka@snow.plala.or.jp

Key words: single-incision laparoscopic surgery, laparoscopically assisted vaginal hysterectomy, learning curve, minimally invasive surgery, uterine myomas myomas and adenomyosis $(1,2)$, usually by placing three trocars through the abdominal wall. To further reduce the invasiveness of traditional laparoscopic procedures, transumbilical singleincision laparoscopic surgery has been gradually developed mainly in general surgery, since Navarra et al introduced the 'one-wound laparoscopic cholecystectomy' technique in 1997 (3). In the field of gynecology, although the single-incision laparoscopic procedure was described in cases of sterilization in the 1970s (4), it has not been well developed to date.

There are several hysterectomy procedures using a laparoscope, such as LAVH and total laparoscopic hysterectomy (TLH), to reduce invasiveness. Total vaginal hysterectomy (TVH) is another less invasive hysterectomy procedure without an abdominal wound. We usually perform LAVH for patients with uterine myomas and adenomyosis since it is less invasive than 'open' total abdominal hysterectomy (TAH), safer than TVH and it requires less complicated laparoscopic procedures than TLH. We applied single-incision laparoscopic surgery to LAVH for 10 cases in July 2009 and considered that it could be undertaken effectively with similar operative results, compared to conventional multiport, multi-incision LAVH (5). Since then, we shifted the conventional laparoscopic procedure to a single-incision one when performing LAVH. To better evaluate this operation, we accumulated cases in which single-incision LAVH was performed for uterine myomas and adenomyosis. We began to apply singleincision LAVH in July 2009 and we planned to perform this procedure in 50 cases until March 2010. Here, our operative outcomes are presented and compared to conventional multiport, multi-incision LAVH cases. We also tried to estimate the learning curve of this method comparing the operative results of the former and the latter cases during the study period.

\section{Materials and methods}

Patient selection. From July 2009 until March 2010 (we started to apply single-incision LAVH in July 2009), 50 female patients underwent single-incision LAVH for uterine myomas or adenomyosis. All single-incision LAVHs were performed by the same operator (S.M.) with written informed consent provided by the patients.

The mean age of the patients was $45.2 \pm 4.0$ years (range 36-53). These 50 patients suffered from one or more of the following symptoms: pelvic pain, hypermenorrhea, dysmenorrheal and compression syndrome, such as constipation 
and incontinence. In all patients, the presence of myomas or adenomyosis was confirmed clinically by transvaginal ultrasonography and computed tomography or magnetic resonance imaging. Patient characteristics and operative results, including operative time, estimated blood loss, weight of resected uterus and additional procedures, were recorded. Additionally, the results of 40 patients who underwent conventional multiport, multi-incision LAVH performed by the same operator from January until June 2009, prior to introducing single-incision LAVH, were also recorded and compared to the single-incision LAVH cases. The mean age of the conventional LAVH patients was $47.3 \pm 6.1$ years (range 35-65) and the indications were also uterine myomas or adenomyosis. Gonadotropin-releasing hormone agonists (GnRHa) were administered pre-operatively when the uterus was larger than a baby's head size, or when complicated by anemia or endometriosis; 17 cases (34\%) in the single-incision LAVH group and 12 cases (30\%) in the conventional LAVH group, respectively $(\mathrm{P}=0.69)$.

To estimate its learning curve, 47 completed singleincision LAVH cases were divided into two groups; the former 24 cases (from July until November 2009) and the latter 23 cases (from December 2009 until March 2010). We compared the operative outcomes between the two groups.

Operative technique. LAVH consists of two main operative procedures; one is a laparoscopic procedure and the other is a vaginal approach. We applied single-incision laparoscopic surgery to the laparoscopic phase of LAVH as we previously reported (5). The vaginal approach was the same as that of the conventional LAVH.

All procedures were performed with the patient under general anesthesia and in the lithotomy position. The Uterine Manipulator $^{\mathrm{TM}}$ (Ethicon, Tokyo, Japan) was inserted into the uterine cavity to manipulate the uterus during the laparoscopic procedure. We completely extroflexed the umbilicus and made a longitudinal skin incision of $\sim 2 \mathrm{~cm}$, bluntly dissecting the subcutaneous tissue to the fascia. As the first trocar, a $5-\mathrm{mm}$ XCEL Bladeless Trocar ${ }^{\mathrm{TM}}$ (Ethicon Endo-Surgery, USA), was inserted with the closed method using a 5-mm 0-degree rigid laparoscope. After insertion, pneumoperitoneum was induced with carbon dioxide $\left(\mathrm{CO}_{2}\right)$ maintained at $8-10 \mathrm{mmHg}$, and the abdominal cavity was explored with a 5 -mm 30 -degree rigid laparoscope. The second and third 5-mm working trocars (Lina Port ${ }^{\mathrm{TM}}$; LiNA Medical, Glostrup, Denmark) were placed through separate fascial punctures within one skin incision in the umbilicus. Two working trocars were inserted inferiorly to the camera trocar site, crossing each other. All three trocars were placed through one skin incision at different fascial sites to create a triangulated arrangement (Fig. 1). Both roticulating and straight forceps were used depending upon the operative findings to create the necessary operative field.

Round ligament, fallopian tube, ovarian ligament and/or vesicouterine excavation peritoneum were cut with a vessel sealing system such as LigaSure $^{\mathrm{TM}}$ (Covidien, MA, USA) and/or electric scissors during the laparoscopic procedure. Then, we shifted to the vaginal approach. Uterine artery and lateral cervical ligaments were cut and the resected uterus was removed transvaginally. The peritoneum and vaginal walls were closed with the vaginal approach. After confirming
A

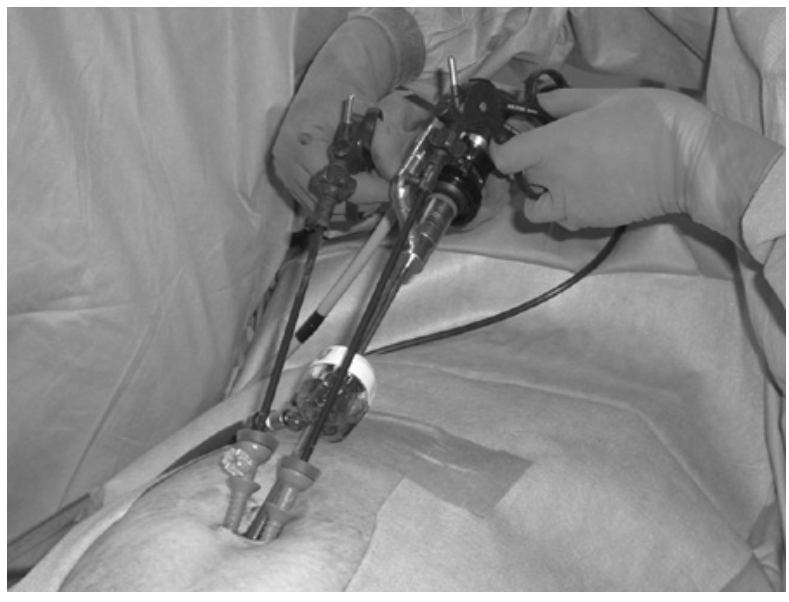

B

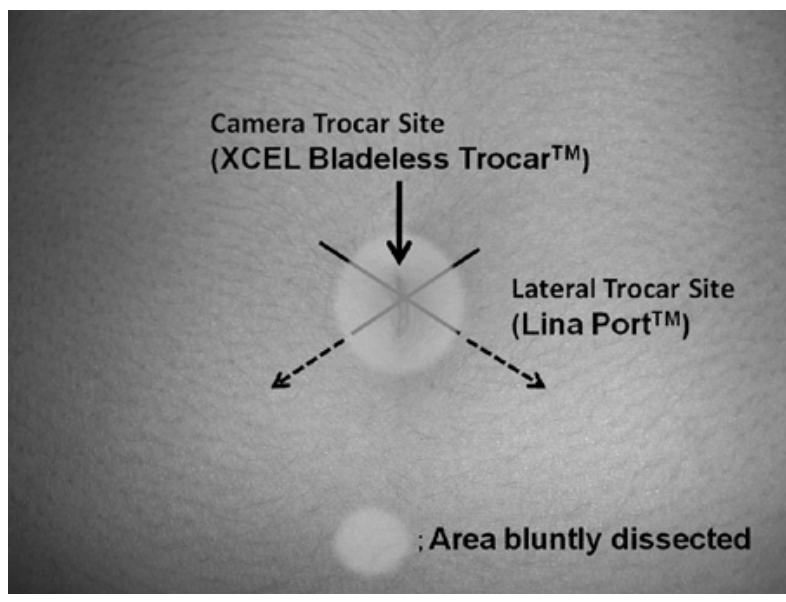

Figure 1. (A) Triangulated arrangement with three trocars used in single-incision LAVH. (B) Schematic image of the umbilicus showing the triangulated arrangement. Multiple fascial punctures were made through one skin incision within the umbilicus. Arrows show the direction of the inserting trocars.

sufficient hemostasis laparoscopically, the fascial incisions were closed with a 0-Vicryl suture. The umbilicus was carefully reconstructed with a 4-0 Vicryl suture, allowing it to be replaced to its original position. As a result, the abdominal wound appeared completely invisible.

Statistical analysis. All results are expressed as the means \pm standard deviation. Operative time was defined as the time from skin incision to time of closure. Comparisons between the two groups were performed using the Mann-Whitney U-test for independent sample for continuous values and the $\chi^{2}$ test or Fisher's exact test for categorical variables. Statistical analysis was performed with SPSS version 10.0 (SPSS, Chicago, IL, USA).

$\mathrm{P}<0.05$ was considered statistically significant and all reported P-values were two-sided.

\section{Results}

Fifty patients underwent single-incision LAVH from July 2009 until March 2010. Before applying single-incision LAVH, 40 patients underwent conventional multiport, multiincision LAVH during the first half of 2009.

Regarding the characteristics of the patients, only body mass index was significantly different between the single-inci- 
Table I. Characteristics of the patients who underwent single-incision LAVH and conventional multiport, multi-incision LAVH.

\begin{tabular}{|c|c|c|c|}
\hline & Single-incision LAVH $(n=50)$ & Conventional LAVH $(n=40)$ & P-value \\
\hline Age, in years & $45.2 \pm 4.0(36-53)$ & $47.3 \pm 6.1(35-65)$ & 0.08 \\
\hline $\mathrm{BMI}$, in $\mathrm{kg} / \mathrm{m}^{2}$ & $22.0 \pm 2.7(16.4-28.4)$ & $23.6 \pm 3.5(17.5-30.5)$ & 0.03 \\
\hline History of vaginal delivery & $42(84 \%)$ & $36(90 \%)$ & 0.60 \\
\hline Indications & & & 0.63 \\
\hline Myoma & $45(95 \%)$ & $38(95 \%)$ & \\
\hline Adenomyosis & $5(10 \%)$ & $2(5 \%)$ & \\
\hline Pre-operative GnRHa administration & $17(34 \%)$ & $12(30 \%)$ & 0.69 \\
\hline
\end{tabular}

LAVH, laparoscopically assisted vaginal hysterectomy; BMI, body mass index; GnRHa, gonadotropin-releasing hormone agonists. Data are expressed as the mean \pm standard deviation (range) or number $(\%)$.

Table II. Operative results and comparison between completed single-incision LAVH and conventional multiport, multi-incision LAVH.

\begin{tabular}{|c|c|c|c|}
\hline & Single-incision LAVH $(n=47)$ & Conventional LAVH $(n=40)$ & P-value \\
\hline Operative time (min) & $65.0 \pm 17.0(37-110)$ & $63.9 \pm 22.5(30-120)$ & 0.51 \\
\hline Estimated blood loss (ml) & $359 \pm 261(46-1,389)$ & $344 \pm 242(46-1,107)$ & 0.83 \\
\hline Weight of resected uterus (g) & $324 \pm 173(86-850)$ & $369 \pm 199(114-950)$ & 0.38 \\
\hline Additional procedures & $13(27.7 \%)$ & $23(57.5 \%)$ & $<0.01$ \\
\hline Salpingo-oophorectomy & 4 & 10 & \\
\hline Adhesiolysis & 9 & 13 & \\
\hline Conversion & $\begin{array}{c}\text { to conventional LAVH } \\
3 / 50(6 \%)\end{array}$ & $\begin{array}{c}\text { to TAH } \\
0\end{array}$ & 0.12 \\
\hline
\end{tabular}

LAVH, laparoscopically assisted vaginal hysterectomy; TAH, total abdominal hysterectomy. Data are expressed as the mean \pm standard deviation (range) or number $(\%)$.

sion and conventional LAVH, as shown in Table I $(22.0 \pm 2.7$ vs. $23.6 \pm 3.5, \mathrm{P}=0.03)$. Table II shows the operative results of single-incision and conventional LAVH. We completed 47 of $50(94 \%)$ single-incision LAVHs in this study. We experienced 3 conversion cases to conventional multiport surgery from single-incision LAVH, and no conversion case to TAH from conventional LAVH $(3 / 50,6 \%$ vs. $0 / 40,0 \%, \mathrm{P}=0.12)$. We did not experience any conversions to TAH from single-incision LAVH. The reasons for conversion were as follows: strong pelvic adhesion in 2 cases and large uterine myomas in 1 case that made it difficult to create the necessary operative field or to manipulate the forceps. Operative time, estimated blood loss and weight of the resected uterus were statistically similar, but more additional procedures, such as salpingo-oophorectomy and adhesiolysis, were required in the conventional LAVH group $(27.7 \%$ in single-incision vs. $57.5 \%$ in the conventional group, $\mathrm{P}<0.01)$. None of the patients required a blood transfusion and no major perioperative complications, such as organ damage, reoperation, surgical site infections or hernias, were encountered in either group.

Upon dividing the 47 completed single-incision LAVH cases into two groups by the period in which the operation was performed, the operative time was significantly shortened during the study period, as shown in Fig. 2 and Table III;

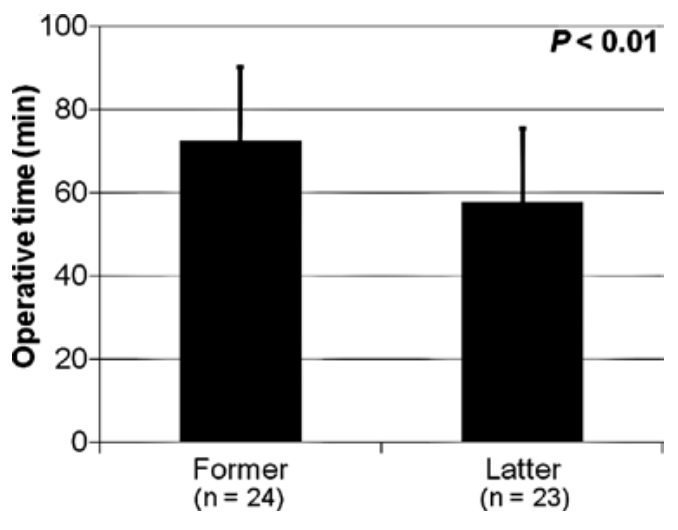

Figure 2. Operative time of the completed single-incision LAVH cases and comparison between the two periods. The former 24 cases were performed from July until November 2009, and the latter 23 cases were performed from December 2009 until March 2010. The difference between the two periods was statistically significant $(\mathrm{P}<0.01)$.

$73.0 \pm 17.6 \mathrm{~min}$ for the former 24 cases vs. $58.0 \pm 12.2 \mathrm{~min}$ for the latter 23 cases $(\mathrm{P}<0.01)$. These results suggest a short learning curve for single-incision LAVH. There was no significant difference between the two subgroups with respect to 
Table III. Operative results of completed single-incision LAVH cases and comparison between the two periods.

\begin{tabular}{lccr}
\hline & Former cases $(\mathrm{n}=24)$ & Latter cases $(\mathrm{n}=23)$ & P-value \\
\hline Operative time (min) & $73.0 \pm 17.6(42-110)$ & $58.0 \pm 12.2(37-85)$ & $<0.01$ \\
Estimated blood loss (ml) & $355 \pm 213(73-909)$ & $363 \pm 312(46-1,389)$ & 0.59 \\
Weight of resected uterus $(\mathrm{g})$ & $349 \pm 203(94-850)$ & $298 \pm 138(86-598)$ & 0.59 \\
Additional procedures & $6(25 \%)$ & $7(30.4 \%)$ & 0.68 \\
Salpingo-oophorectomy & 2 & 2 & \\
Adhesiolysis & 4 & 5 & \\
\hline
\end{tabular}

LAVH, laparoscopically assisted vaginal hysterectomy; Data are the means \pm standard deviation and the range or number (\%). The former 24 cases were performed from July until November 2009, and the latter 23 cases from December 2009 until March 2010.

estimated blood loss, weight of resected uterus and additional procedures (Table III).

\section{Discussion}

The main operative results, including operative time, estimated blood loss and weight of resected uterus, were compared and there was no significant difference between single-incision LAVH and conventional LAVH. In addition, we first showed an even shorter learning curve for singleincision LAVH based on our operative results.

First, there was no significant difference between singleincision LAVH and conventional LAVH when the main operative results were compared. The main procedure of $\mathrm{LAVH}$ is the vaginal approach and this is the same in both single-incision and conventional LAVH. Therefore, operative time and estimated blood loss were not influenced by the laparoscopic procedure when performing single-incision LAVH. These laparoscopic procedures through one skin incision within the umbilicus may be performed with similar operative results to those of the conventional multiport, multiincision LAVH. Through these outcomes, it may be possible to consider the indication of single-incision LAVH to be same as that of conventional LAVH. In addition, approximately $30 \%$ of the patients were administered GnRHa before surgery in this study. GnRHa administration is helpful to extend the indications for single-incision LAVH.

Second, we first showed an even shorter learning curve for single-incision LAVH. Although another comparable operative outcome of single-port-access LAVH has been reported (6), the learning curve of this procedure has not been evaluated before. Completing a new surgical procedure requires appropriate training and experience. It is expected that the mean procedure duration will be gradually shortened with increasing surgical experience $(1,7)$. It has been reported and estimated that a surgeon will be able to establish proficiency after having performed more than 50 conventional LAVHs (8). However, in this study the operative time was significantly shortened during a period of 9 months (less than 50 cases) without special equipment or training. Therefore, there is an even shorter learning curve for single-incision LAVH based on our operative results. This is because the techniques necessary for conventional multiport LAVH are identical to those required to perform and complete the single-incision laparoscopic procedures. Also, difficulties are more likely to be encountered very early in the learning phase with respect to single-incision LAVH.

During this study period, we experienced three conversions from single-incision to conventional multiport LAVH. Although we achieved a high success rate with both singleincision (94\%) and conventional LAVH (100\%), surgeons should not persist in completing laparoscopic procedures in single-incision LAVH, as single-incision procedures can easily be converted to a conventional multiport LAVH in the event problems arise. As a result, severe perioperative complications, such as blood transfusion and adjacent organ damage are avoided.

In the development of the single-incision surgical technique, many different choices and combinations of operative devices, including trocars and forceps, for gynecologic surgery have been proposed $(9,10)$. For the trocars, the combination of the XCEL Bladeless Trocar ${ }^{\mathrm{TM}}$ (camera trocar) and the Lina Port ${ }^{\mathrm{TM}}$ (lateral trocars) was chosen to minimize the conflict between the operative instruments and the camera. A comparison and evaluation of the operative outcomes with the different choices and combinations of surgical devices, such as trocars and forceps, during the operation are warranted. As for the application to other gynecologic diseases, we have already applied single-incision laparoscopic surgery to various ovarian procedures such as salpingo-oophorectomy and cystectomy, as well as the additional procedures of single-incision LAVH. A comparison of the single-incision technique, as applied to these different procedures, with conventional multiport methods is also warranted as it would be possible to evaluate its versatility as well.

We presented data for a sequential comparison, but the conventional LAVHs in this study were performed before applying the single-incision surgical technique. This factor may have introduced certain biases, particularly as it relates to surgical experience and the learning curve. Therefore, it is necessary to provide a comparison of cases performed concurrently. To further establish the level of reliability for single-incision LAVH, more cases and randomized controlled trials are required to precisely evaluate the efficacy and safety, including complication rates.

In conclusion, single-incision LAVH can be performed as effectively as conventional multiport LAVH with a short learning curve. It may be a promising alternative method for 
the treatment of certain patients with uterine myomas and adenomyosis as even a less invasive gynecological operation is required without visible scars.

\section{References}

1. Seow KM, Tsou CT, Lin YH, Hwang JL, Tsai YL and Huang LW: Outcomes and complications of laparoscopically assisted vaginal hysterectomy. Int J Gynaecol Obstet 95: 29-34, 2006.

2. Drahonovsky J, Haakova L, Otcenasek M, Krofta L, Kucera E and Feyereisl J: A prospective randomized comparison of vaginal hysterectomy, laparoscopically assisted vaginal hysterectomy, and total laparoscopic hysterectomy in women with benign uterine disease. Eur J Obstet Gynecol Reprod Biol 148: $172-176,2010$.

3. Navvara G, Pozza E, Occhionorelli S, Carcoforo P and Donini I: One-wound laparoscopic cholecystectomy. Br J Surg 84: 695, 1997.

4. Wheeless CR Jr: Outpatient laparoscope sterilization under local anesthesia. Obstet Gynecol 39: 767-770, 1972.

5. Koyanagi $\mathrm{T}$ and Motomura S: Transumbilical single-incision laparoscopic surgery: application to laparoscopically assisted vaginal hysterectomy. Arch Gynecol Obstet 283: 305-309, 2011.
6. Kim TJ, Lee YY, Cha HH, et al: Single-port-access laparoscopic-assisted vaginal hysterectomy versus conventional laparoscopic-assisted vaginal hysterectomy: a comparison of perioperative outcomes. Surg Endosc 24: 2248-2252, 2010.

7. Chapron C, Querleu D, Bruhat MA, Madelenat P, Fernandez H, Pierre F and Dubuisson JB: Surgical complications of diagnostic and operative gynaecological laparoscopy: a series of 29,966 cases. Hum Reprod 13: 867-872, 1998.

8. Hwang JL, Seow KM, Tsai YL, Huang LW, Hsieh BC and Lee C: Comparative study of vaginal, laparoscopically assisted vaginal and abdominal hysterectomies for uterine myoma larger than $6 \mathrm{~cm}$ in diameter or uterus weighing at least $450 \mathrm{~g}$ : a prospective randomized study. Acta Obstet Gynecol Scand 81: 1132-1138, 2002.

9. Lee YY, Kim TJ, Kim CJ, et al: Single-port access laparoscopicassisted vaginal hysterectomy: a novel method with a wound retractor and a glove. J Minim Invasive Gynecol 16: 450-453, 2009.

10. Lim MC, Kim TJ, Kang S, Bae DS, Park SY and Seo SS: Embryonic natural orifice transumbilical endoscopic surgery (E-NOTES) for adnexal tumors. Surg Endosc 23: 2445-2449, 2009. 